\title{
Live and Let Live: The Remarkable Story of HEK293 Cells
}

\author{
How a single cell line lit the spark that turned gene therapies from theoretical possibility to therapeutic \\ reality
}

All scientists dream of making an impact. Few can look back and identify a single experiment that led to the creation of an entire scientific field.

When Frank Graham set out to transform human embryonic kidney (HEK) cells with adenoviral DNA in early 1973 , he was trying to understand why some adenoviruses caused cancer and some didn't. He wasn't trying to set in motion the creation of a new field of medical research, with a multibillion-dollar market value. But that's exactly what he did.

Frank had left Canada three years earlier to begin his postdoctoral research with Professor Alex van der Eb at the University of Leiden in the Netherlands. Until then, he hadn't been particularly interested in adenoviruses, but finding a postdoctoral supervisor working in that area changed his mind.

"I decided to investigate the correlation between low GC content in adenoviral DNA and oncogenicity," Graham recalls. "At that time, nobody knew much about how or why cancers started, so using oncogenic viruses to transform normal cells into cancerous cells in culture seemed a good in vitro model for tumour induction. Perhaps it sounds naïve now, but given the little we understood back then, seeking to understand what made

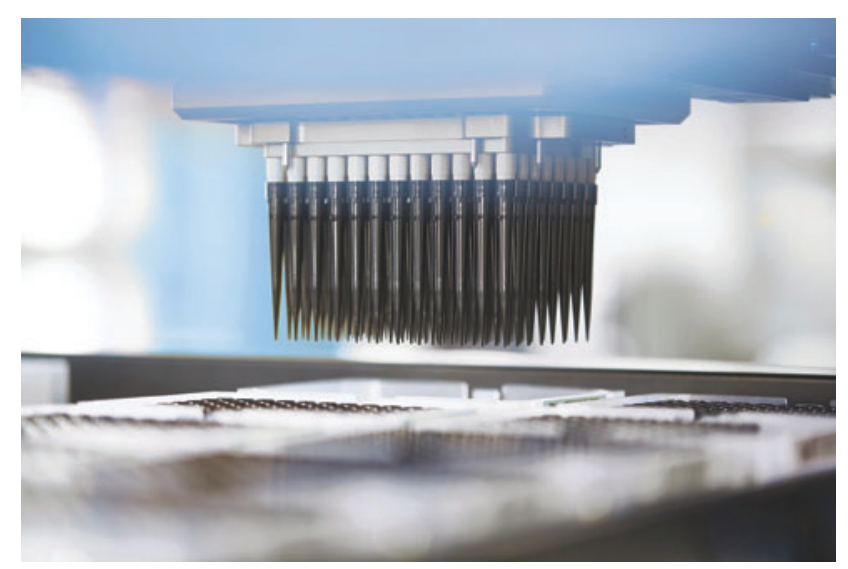

Industry leading technology and cutting edge automation is central to OXGENE's cell line development workflow. some viruses able to initiate this transformation while others couldn't seemed a reasonable way to go about understanding the root causes of cancer."

Graham made his first attempt to transform HEK cells in 1972. He transfected 40 dishes of cells, but only one produced even a single transformed colony, and even that subsequently failed to grow. He had more success in early 1973 , identifying a pair of transformed colonies. He nurtured these such that one began to grow, albeit slowly. After six months, he had grown sufficient cells to be able to freeze a few vials. "The cells grew slowly - doubling once every 7-10 days - for nearly a year." Then disaster approached.

\section{Back from the brink: an immortal cell line emerges}

The cells entered a classical crisis and failed to grow for 2-3 months for unknown reasons. Years later, Graham's spouse found a likely explanation.

"My wife, Silvia Bacchetti, discovered that pre-crisis HEK293 cells didn't express telomerase," Graham says. The telomerase enzyme prevents telomeres (the repetitive DNA at chromosome tips) from shortening with every cell division. "Without telomerase the telomeres get shorter and shorter with every division until eventually they reach a critical length and the cell dies."

Very few cells survived this crisis. "Silvia's research demonstrated that these post-crisis cells did express telomerase, which suggested that the transition to and through crisis selected for cells producing this enzyme, and potentially other growth-promoting genes too," says Graham. Whatever the explanation, after the crisis passed, the cells' growth rate sped up, doubling every 2-3 days.

Graham had finally created an immortal, adenovirustransformed human cell line.

\section{Laying the foundations of gene therapy}

Graham returned to Canada, joining McMaster University in Ontario, where he continued his research into adenoviruses, focusing first on understanding the molecular biology of cell transformation and later the

Sponsored content is not written by and does not necessarily reflect the views of the Human Gene Therapy editorial staff. This sponsored content feature has been produced with funding support from OXGENE. 


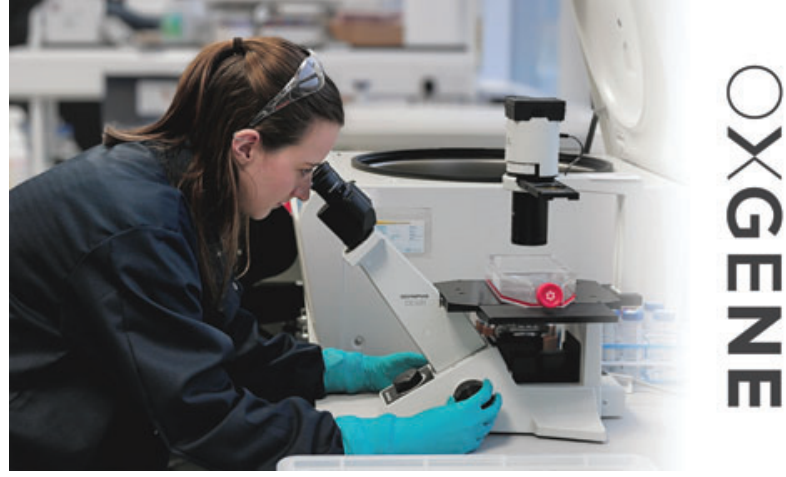

OXGENE's scientists adapted a parental pool of HEK293 cells to grow in different media, before screening thousands of single cell clones for optimum AAV production.

development of adenoviruses as vectors for mammalian gene transfer.

Graham's HEK293 cells held an advantage for this research; their transformation with adenoviral DNA meant that they could support the growth of adenoviruses altered so that they could no longer self-replicate. An altered virus can't grow outside HEK293 cells or other cell types that support adenoviral replication. However with the support of the missing viral DNA that's now incorporated into the cells' genome, the virus can continue to grow. The adenovirus can also be further altered to include human gene products (such as normal copies of disease genes). Once sufficient quantities of the virus have grown in HEK293 cells, they can be transferred into patients with genetic disease. There they will express the correct, or therapeutic, copies of the gene, so counteracting the effect of the original mutation or treating a disease, but can't replicate to cause disease themselves.

This is the fundamental premise of gene therapy and accounts for much of the commercial interest in HEK293 cells.

\section{A cure and a flaw}

Until the late ' 80 s/early ' 90 s, most research using Graham's cells was academic, as he shared them freely within the academic community. But soon, companies began contacting him about commercial use of the HEK293 cells.

Although early days for gene therapy, by the mid ' 90 s the commercial potential was clear. Researchers believed that it would eventually lead to a cure for most genetic diseases. At the time, almost all gene therapy research involved adenoviral vectors. Georg Roth, principal at Roth Bioprocess Consulting and former Senior Scientist at Berlex (an early licensee of HEK293 cells) said adenovirus vectors were "the workhorse of gene therapies". They were easy to handle and had a good safety profile but could only be manufactured using HEK293 cells.
"Unfortunately," Roth continues, "these cells had a serious flaw." HEK293 cells express the adenoviral gene $E 1$, which enables adenoviruses missing this gene to grow. But when Graham initially transformed the cells, molecular biology techniques were not particularly advanced. "The fragment of viral DNA in 293 cells comprises sequences from the extreme end of the genome and extends across $\mathrm{E} 1$ and beyond." The cells therefore contained adenoviral DNA sequences flanking the viral genes that are typically deleted in adenoviral vectors. "This overlap allows homologous recombination to occur."

Thus in some cases, the replication incompetent adenoviral vector could kick out the therapeutic gene it carried and re-incorporate the E1 gene from the HEK293 cells, resulting in a wildtype virus. This presented a huge safety concern for gene therapies. Not only might a patient receive the therapeutic gene they need but also potentially an adenoviral infection they don't. Alternative viral vectors, notably adenoassociated virus (AAV) and lentiviral vectors, offer a significant improvement as they don't have the same problem with homologous recombination.

\section{The fall of the adenoviral vector for single gene disorders}

By now, it was becoming clear that adenoviruses might not be the holy grail of gene therapies after all. They provoke an immune reaction and don't integrate the therapeutic gene into the target cell, so can only deliver a transient response, not a long-term cure for genetic disease. A critical set-back for adenoviral-based gene therapies came in 1999, with the tragic death of teenager Jesse Gelsinger in a clinical trial at the University of Pennsylvania that aimed to correct an X-linked genetic disease of the liver.

All adenovirus-based gene therapy trials were immediately put on hold, and for years it seemed that the industry would never recover. But scientists realised that it was important to be open with the public about the challenges of gene therapies in order to progress an extremely promising area of medical research.

"At this point we set up the British Society of Gene Therapy", says Len Seymour, Professor of Gene Therapies at the University of Oxford. "We knew that it was important to engage with the public about what had happened and focus on the potential benefits that might still be achieved."

\section{Establishing the potential for gene therapies}

Seymour highlighted three major goals with gene therapies two decades ago. First, oncolytic (cancer killing) viruses. "That - alongside using adenoviruses 
as a vector for vaccines - is where adenoviruses are coming back into their own again now." Graham seconds this sentiment and adds, "Personally, I think vaccine development is the most important application for adenoviral vectors." Second, gene supplementation therapies - the traditional gene therapy or replacement idea. Third, cell therapies, such as the new CAR-T therapies. "In cell therapies you take cells from the patient, modify them in some way and then inject them back in."

By 2010, the renewed potential of gene therapy was clear, and HEK293 cells were still central to manufacturing the viral vectors most commonly used to deliver the replacement gene. In Oxford, scientists at the University's Clinical Biomanufacturing Facility adapted suspension HEK293 cells to serum free suspension culture. Graham's original HEK293 cells grew in adherent culture, which means they stick to the bottom of a cell culture dish. This is fine in most cases (and is how cells usually prefer to grow, because they can maintain close contact with their neighbours, and mimic the formation of tissues within an organism). But for gene therapies that need very large numbers of cells to produce the quantity of virus required for clinical manufacture, the physical space required to culture this number of adherent cells becomes a significant limitation. Adapting cells to grow in suspension is a key element of scaling gene therapies for commercial and clinical manufacture. Adapting the cells further to grow without animal serum is important to take cells into clinical production.

\section{Scalable technologies for gene therapy manufacture}

By 2016, the gene therapy market was back on track in all three areas that Seymour had predicted decades

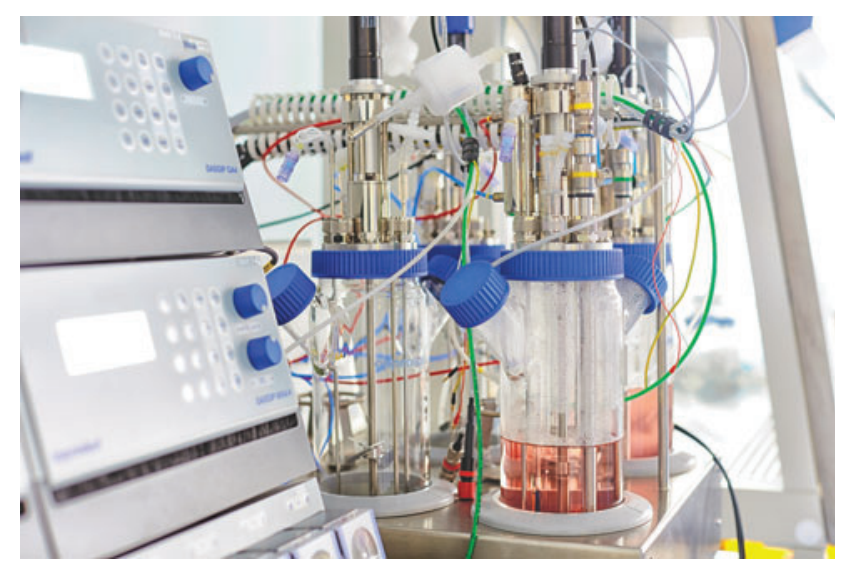

OXGENE's ambition is to pioneer the development of carefully optimised technologies for scalable, cost effective and highquality gene therapy manufacture. ago. Adenoviruses are now widely used as oncolytic viruses, and as vaccines for a range of diseases, but are little used for gene supplementation therapies. There, AAV and lentiviral vectors are far more in vogue, and both are also produced using HEK293 cells.

Around this time, Oxford Genetics - now OXGENE ${ }^{\mathrm{TM}}$ licensed the cells from Oxford University. Their scientists wanted to see whether they could take this cell line, which had been the backbone of the global gene therapy market since its inception, and optimise it to maximise viral yield.

Senior Group Leader, Qian Liu, says: "One of the major challenges of gene therapy nowadays is scaling manufacture. At the moment, it's difficult - and very expensive - to produce the amount of virus necessary to treat a patient, especially one with a systemic disease. Those costs are unfortunately passed down to the patient, or the health providers. Right now, a course of gene therapy treatment can cost over $\$ 2$ million. That's clearly unsustainable. We want to find a way to make gene therapies more cost effective, and scalable manufacture is a big part of that."

HEK293 cells are central to the entire OXGENE process. "One of our aims is to enable efficient manufacture of AAV, for example serotype AAV5." OXGENE scientists adapted the parental cell line to grow in a variety of media types, which supply nutrients, energy and growth factors needed to survive and function. Different types of media can alter the cells' growth rate and/or the gene expression profile. For example, one type of media proved optimal for AAV5 production.

"Next we sorted the media-adapted parental cell population into single cells and selected the clones that grew fast and produced the most rAAV5," Liu continues. "This same clone was also good at producing lentivirus too, and a few other AAV serotypes. Once we'd properly established our clonal cell line, we started the process of GMP banking it."

GMP - or Good Manufacturing Process - certification is a process by which cells are manufactured in a controlled and regulated manner, and tested extensively for any contaminants and pathogens. This certification is essential to use the cells in any therapeutic application or clinical trial.

"We're extremely excited to have GMP-banked our optimised HEK293 clonal cell line," Liu says. "I love the idea that we can trace these cells all the way back to Frank's experiment in 1973. It's funny to think that without these cells, the gene therapy field might never have opened up the way that it has. Now we hope that our cells will be central to the next part of the process too - making gene therapies scalable, cost effective, and accessible to everyone who needs them." 